\author{
MITSUBISHI ELECTRIC RESEARCH LABORATORIES \\ http://www.merl.com
}

\title{
Building and Sharing Digital Group Histories
}

\author{
Shen, C.; Lesh, N.B.; Vernier, F.; Forlines, C.; Frost, J.
}

TR2002-07 November 2002

\begin{abstract}
Organizations, families, institutions evolve a shared culture and history. In this work, we describe a system to facilitate conversation and storytelling about this collective past. Users explore digital archives of shared materials such as photographs, video, and text documents on a tabletop interface. Both the software and the interface encourage natural conversation and reflection. This work is an application of our ongoing research on systems for multiple, co-present users to explore digital collections. In this paper, we present a case study of our own group history along with the software extensions developed for this scenario. These extensions include methods for easily branching off from and returning to previous threads of the exploration, incorporating background contexts that support a variety of view points and flexible story sharing, and supporting the active and passive discovery of relevant information.
\end{abstract}

ACM Conference on Computer Supported Cooperative Work (CSCW)

This work may not be copied or reproduced in whole or in part for any commercial purpose. Permission to copy in whole or in part without payment of fee is granted for nonprofit educational and research purposes provided that all such whole or partial copies include the following: a notice that such copying is by permission of Mitsubishi Electric Research Laboratories, Inc.; an acknowledgment of the authors and individual contributions to the work; and all applicable portions of the copyright notice. Copying, reproduction, or republishing for any other purpose shall require a license with payment of fee to Mitsubishi Electric Research Laboratories, Inc. All rights reserved.

Copyright (C) Mitsubishi Electric Research Laboratories, Inc., 2002

201 Broadway, Cambridge, Massachusetts 02139 



\title{
Sharing and Building Digital Group Histories
}

\author{
Chia Shen Neal B. Lesh \\ Frederic Vernier \\ MERL - Mitsubishi Electric Research Laboratories \\ 201 Broadway, Cambridge MA 02139 USA \\ 1-617-621-7500 \\ \{shen, lesh, vernier, forlines\}@merl.com
}

Clifton Forlines

Jeana Frost

\begin{abstract}
Organizations, families, institutions evolve a shared culture and history. In this work, we describe a system to facilitate conversation and storytelling about this collective past. Users explore digital archives of shared materials such as photographs, video, and text documents on a tabletop interface. Both the software and the interface encourage natural conversation and reflection. This work is an application of our ongoing research on systems for multiple, co-present users to explore digital collections. In this paper, we present a case study of our own group history along with the software extensions developed for this scenario. These extensions include methods for easily branching off from and returning to previous threads of the exploration, incorporating background contexts that support a variety of view points and flexible story sharing, and supporting the active and passive discovery of relevant information.
\end{abstract}

\section{Keywords}

Group history, digital story sharing, single-display groupware.

\section{INTRODUCTION}

One of the best parts of life is sharing experiences with others, whether with family over dinner, friends we see occasionally, relatives who live far away, or colleagues with whom we work. People often use records of their past, such as photographs, videos and electronic documents, to help tell their stories. Recent advances in technology have made it possible to easily amass large collections of digital recordings of our daily lives. These media offer opportunities for new story sharing experiences. The Personal Digital Historian (PDH) project is an ongoing effort to help people construct, organize, navigate, and share digital collections in an interactive multi-person conversational setting $[16,22]$.

Permission to make digital or hard copies of all or part of this work for personal or classroom use is granted without fee provided that copies are not made or distributed for profit or commercial advantage and that copies bear this notice and the full citation on the first page. To copy otherwise, or republish, to post on servers or to redistribute to lists, requires prior specific permission and/or a fee.

CSCW'02, November 16-20, 2002, New Orleans, Louisiana, USA.

Copyright 2002 ACM 1-58113-560-2/02/0011 ..\$5.00.
The overall goal of PDH includes helping people both share and reflect on either individual or collective experiences. A single user might use PDH to reflect on her past experiences, or multiple users might use PDH while relating their experiences to each other. The digital collections might pertain to one user or to a larger group to which the user(s) belong. In this paper, we focus on multi-person usage of PDH for data that relevant to a group. We use the term "story sharing" instead of storytelling to emphasize our support for the communication and conversation among the participants in a group, rather than the performance of the storyteller.

Groups, such as families, clubs, and companies, can use digital collections of recordings to communicate stories from generation to generation, help new members integrate into the organization, and enhance the sense of culture and community within the organization. Additionally, a methodological advantage of investigating group histories is that we are able to get a large number of people to participate in a single application scenario. Group histories raise interesting challenges, such as how to represent different points of view of the same event.

We developed a group history of our own research lab as the first application of PDH. This case study provided information on the contents that are important for a digital group history, and the features that are important for exploring the content. The study was composed of exploratory research, development, and testing. We performed an initial set of interviews with senior members of the lab to establish the key events of the lab history. Simultaneously we constructed and annotated our initial collection of data about the lab that illuminated additional requirements for PDH. Finally, we performed a preliminary user study in which pairs of members of our lab used PDH to explore the group history we constructed.

Our paper is organized as follows. We first describe our interviews and construction of our group history for our lab. We then briefly describe the basic PDH system for annotating digital data and exploring digital collections. Next, we present new features designed to specifically support multi-person interaction with group histories. We then describe our user study, and conclude with a discussion of related and future work. 


\section{CASE STUDY: A GROUP HISTORY OF OUR LAB}

We focused our investigation into building and sharing group histories by thinking about what a group history of our own research lab should include.

The first step we took was to consult with a local memoir writer. He described two effective methods he uses to construct memoirs for his clients that are relevant for constructing group histories. One method is to interview various people, e.g., siblings, friends or collaborators, who have shared experience in the same event. The second method is to show pictures and images to his clients. This achieves two goals. One is to jog the client's memory for the relevant events. And the other is to make it easier for a client to elaborate and articulate his or her stories. Sometimes, the photos or pictures used are not even the personal photos of the client. They can be newspaper clippings or magazine images.

Encouraged by his comments, we interviewed members of the lab to determine the major themes and stories from the lab's history, and how people differ in their versions of the same stories. We also collected about 1000 pictures dating back over the last 10 years. The pictures were not evenly distributed but were concentrated on certain events, primarily official events, parties and picnics. The pictures show who has worked at the lab over the years, but not very much of what has occurred here or what day-to-day life was like. We also collected about 250 technical report abstracts produced by the researchers here. These give a good sense of what people have been working on at various points in the lab's history.

The interviews revealed that much of the history of our lab currently exists in people's heads rather than in documents. We performed three interviews of senior lab members, after a pilot interview on one of the co-authors of this paper. None of the interviewee's needed much prompting to tell their version of the history of the lab. Several events were described by all the interviewees, including the founding of the lab and the development of some of the largest projects that came out of the lab. The interviewees told their own version of the lab's story, emphasizing different aspects of what happened. In general, background context such as economic and political events that were external to the lab figured importantly in the telling of the lab's activities. Several of the interviewees described our lab by comparing or contrasting it to other labs, including ones that started around the same time but no longer exist. Finally, it was also clear that the current conditions and perspective of the lab is influencing the description of the past. For example, the lab is currently putting a lot of energy into technologytransfer, and so it is easy to describe the lab in terms of it's past technology-transfer efforts. Here the context in which the stories were told was important.

Additionally, the interviewees were encouraged to draw timelines reflecting their perception the lab history. The timelines varied dramatically in both style and contents from person to person. An example timeline is shown in Figure 4.

The insights we gained from this case study were:

- The system should provide support for differences in the way people share stories. Storytelling is subjective and we can represent personal style, biases and discrepancies in the design of the interface. For example, an individual may compress or elongate the view of time acc2ording to his/her own memory.

- A story-sharing system designed to support group histories must support flexible narrative, rather than rigid, pre-authored stories. The contents must contain enough structure so that new members of the group can understand and re-tell the stories themselves, but not so much structure that people are locked into one way of describing the relevant events.

- Because stories occur with a cultural and historical context, the system should provide facilities to bring out background and contextual information, e.g., relevant news clippings, to assist the user to interactively reflect on and share past experiences of the group.

\section{OVERVIEW OF PDH}

The PDH prototype is designed as a single-display multiuser piece of furniture containing a circular tabletop display, shown in Figure 1. The PDH software is based on a set of general-purpose techniques for multi-person document sharing on a horizontal, circular interface presented in [22]. The documents are positioned using a polar coordinate system, rather than the Cartesian system used for rectangular displays. Users can re-orient individual documents or rotate the entire display. The system provides two different fisheye deformation techniques for automatically re-sizing documents, and to allow for efficient management of large numbers of documents. Additionally, documents can be individually re-sized by the users. Finally, the system provides a variety of techniques to allow people to manage the construction and layout of hierarchical groupings of documents (see Figure 2).

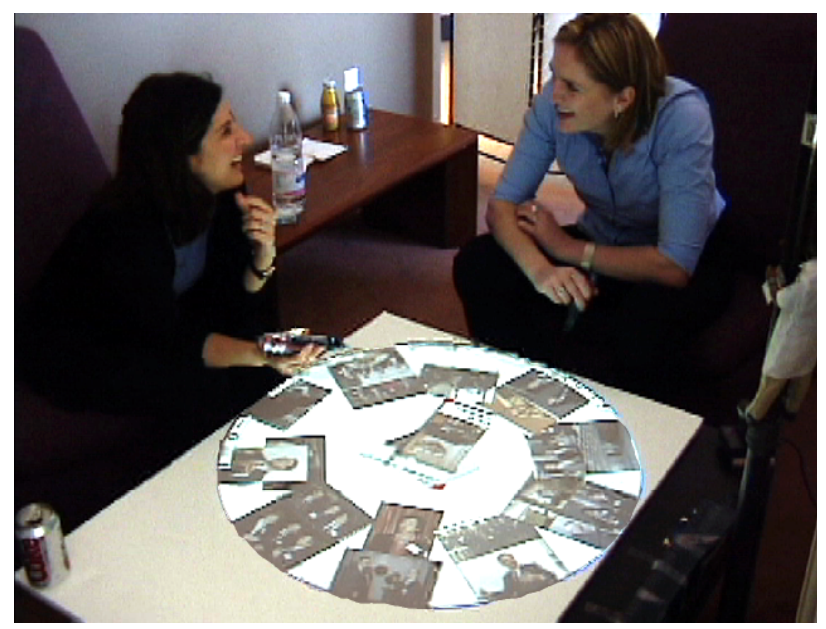

Figure 1: The PDH table. 
We are in the process of constructing the initial PDH prototype. The PDH user interface is being implemented in Java on Windows 2000. Our digital photo database is based on Microsoft Access ${ }^{\mathrm{TM}}$ Database. The PDH interface translates user's visual requests into SQL commands. A tabletop display with top projection onto a standard whiteboard as shown in Figure 1 is currently used as the physical PDH table. Two Mimio [12] styluses are used as input devices for the user experiments reported in this paper.

\section{The Four W's of Document Organization}

Creating a new type of interface requires addressing many issues. One of our primary focuses is on developing content organization and retrieval methods that are easy and understandable for the users, and can be used without distracting them from their conversation. Rather than the folder\&file mechanisms used by conventional document systems, the users of PDH can annotate, organize and query the digital collection of documents around four questions essential to storytelling: who?, when?, where?, and what? Control panels located on the perimeter of the user interface contain four buttons corresponding to these alternatives for organizing information.

When the user presses the "Who" button, PDH displays a portrait of all of the people who appear in the database. The portrait can be specified by the user, or will be chosen automatically. The view initially displays all portraits in black and white. As described below, the users can form queries by selecting or deselecting people's images. Selected portraits are shown in full color. The Who button on the control panel also lists the names of the people that have been selected as shown in Figure 2. This both reminds users of what the current selections are, and allows them to easily retract these selections by touching the names on the button.

Of course, all the people in the database might not fit on the screen at once. Using the generic document-sharing techniques described above, users can form groups of documents, such as putting all of ones work colleagues in a single group, or further subdivide that group into smaller groups.

The What view closely resembles the Who view, but allows users to organize and query their photos based on the objects and events recorded in those pictures, rather than the people who appear in them. The users can chose an image to represent the object or event, and can select and deselect the events and objects in the same manner as selecting or deselecting people in the Who view.

When a user presses the "Where" button, PDH displays a map of the world. Every picture in the database that is annotated with a location will appear as small thumbnail at its location as shown in Figure 3. The user can pan and zoom in on the map to a region of interest, which increases the size of the thumbnails. The user can also select regions of interest. By touching an icon on the control panel, a user essentially turns their stylus into a "freeform stroke" tool [15], which can then be used to identify a region on the map.

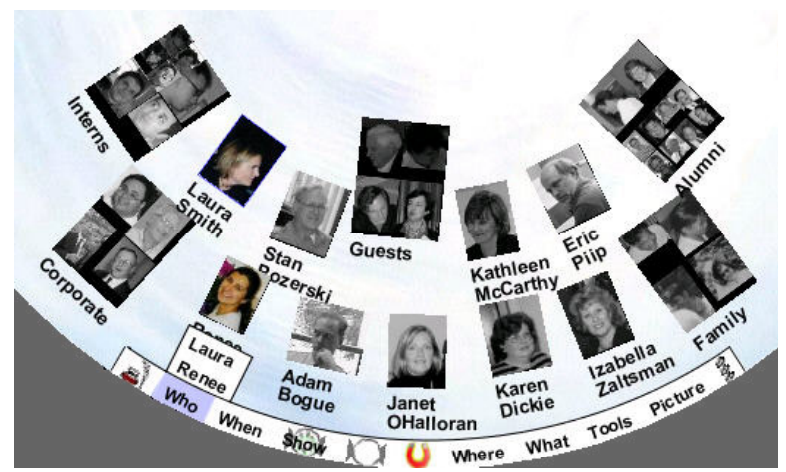

Figure 2: A close-up view of the Who view.

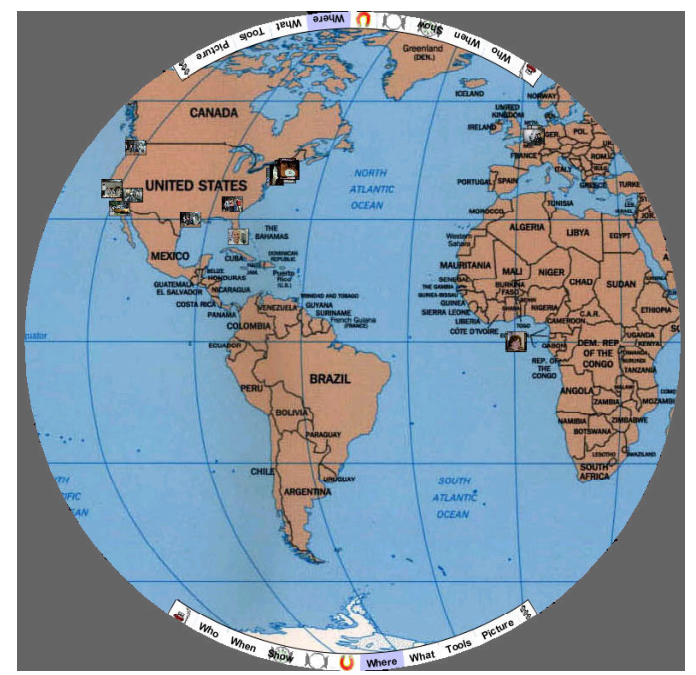

Figure 3: The Where view with geographic registration of photos.

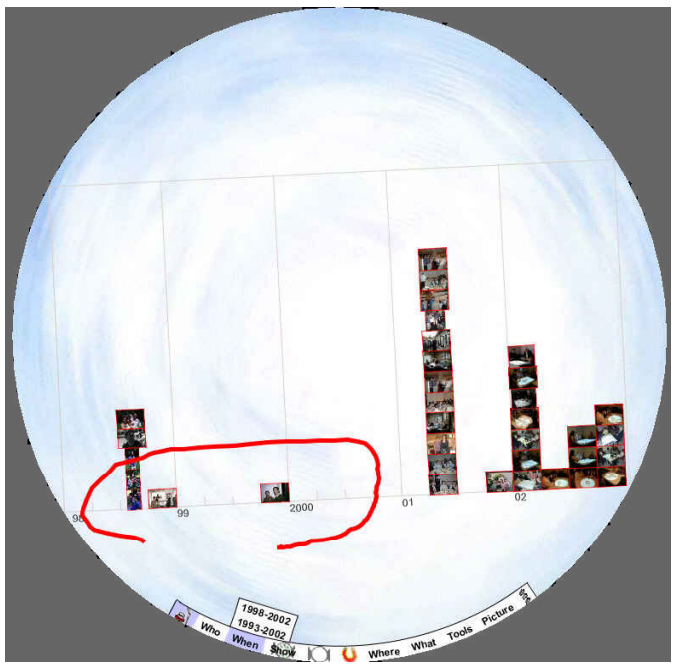

Figure 4: The When view with in-place freeform stroke input to mark region of interest.

By pressing on the When button, the user can indicate to the system to retrieve the pictures to be viewed by the time they 
were taken along a linear timeline as shown in Figure 4. A user can press on any photo in the calendar view to enlarge that photo. The users can pan and zoom into region of interest they select.

\section{Implicit Query Formulation and The "Show" Button}

Studies have shown that Boolean logic is difficult for novices to grasp. Any successful content retrieval system needs to address this difficulty. In PDH, the users' selections in the four views described above are automatically combined by the PDH system to allow the users to easily form rich Boolean queries. More precisely, the selections made in any of the four views will result in a subset of the images to be highlighted and prominently displayed in other views. The semantics of selecting people, for example, is to tell PDH to highlight only those images containing at least one of the selected people. For example, if a user selects two friends in the Who view, and then switches to the Where view, then they would see where they have traveled with either of these friends by observing where the highlighted pictures appear on the map. If the users select several people in the Who view, and several objects in the What view, then PDH will highlight only pictures that contain at least one of the selected people and at least one of the objects. We found this AND/OR semantics to be the most natural way to quickly form queries. Similarly, the users can select regions of interest in time or space with the When or Where views.

There is a fifth view available to users in which photos are not organized by any particular dimension. Pressing the "Show" button on the control panel tells PDH to retrieve and display the photos in the user's collection that match all the constraints the users have specified by selecting items or regions of interest in the four $\mathrm{W}$ views described above. An example of this view is in Figure 5 and Figure 10. Thus, if a user wants to see all and only pictures of her grandmother taken in Paris in 1995, then she can specify those constraints in the Who, Where, and When views, and then press the Show button. PDH will then switch to the "Free Space" view and display the relevant pictures. Some initial visualization and layout techniques for documents and images for this Free Space view have been reported in [22].

\section{NEW PDH FEATURES FOR GROUP HISTORIES}

The following four features grew out of the current project for creating a group history for our research lab. While they are applicable to any of the usage scenarios for $\mathrm{PDH}$, we have found them especially well suited to multi-person exploration of group data. (The third feature, association generation, existed in PDH prior to this project but was further developed during the course of it.)

\section{Image-based Visual Book-Marking}

During a conversation, people often branch out to different topics and threads, and then come back to some previous discussion point. Current user interface technology typically keeps a linear history of some sort with text-based book marking, and/or a back button. We propose an image-based book marking function which records both the contents retrieved from the database plus the visual layout as individual objects for later recall.

Here we allow the user to choose an item on the tabletop as a landmark (e.g., an image or a document), which will be used as an entry in the PDH bookmark method, and can then be memorized by the user for easy re-use. The advantage of this image-based bookmark technique is analogous to how people remember places where they have traveled to by noticing landmarks. We have chosen to not use a thumbnail screenshot of the entire display as the bookmark because, when zoomed out, all the displays look the same visually as a colored blob. What is really important in a bookmark is the differentiation of the individual memorable entries.

We provide a drag\&drop operation of any document to the bookmark area. Although the main PDH content display area is circular, we actually use a rectangular table top since the projected display is rectangular. This turns into an advantage because we can use the rest of the rectangular area outside the circular interface (the black area in Figures 2 - 10) as private space for users. Figure 5 shows an example of this image-based book-marking function. The small photo on the lower left side of Figure 5 is moved to the private space as the bookmark by the user.

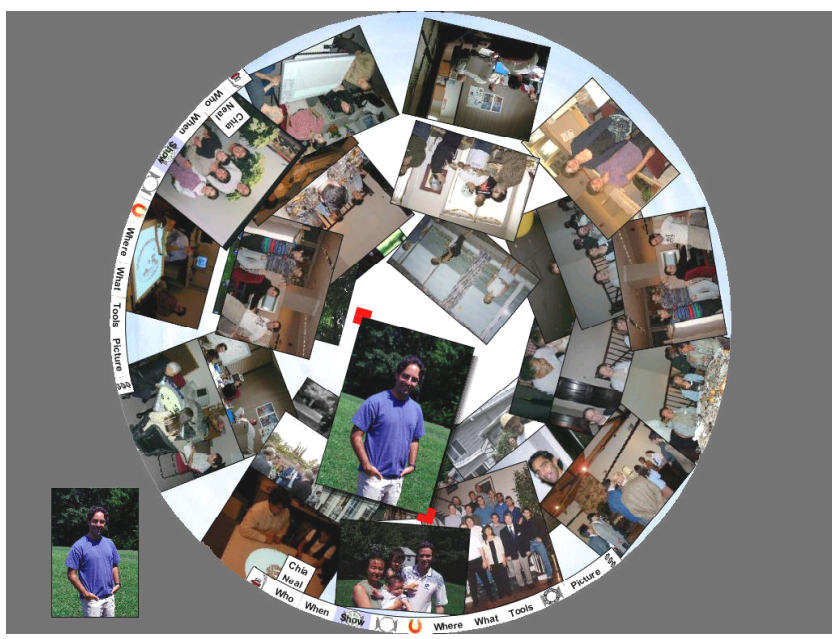

Figure 5: Free Space view with Image-based book marking.

\section{Contextual Images}

Events and stories are framed by context including politics, economy, sociology, art and literature [23]. Event and stories also draw meanings from their context. The importance of background context is evident from our case study interviews described in Section 2.

We have added a mechanism to PDH to allow users to define new contexts. A context is a background image and some method for mapping some or all of the documents in the PDH database to locations on that image. This mapping can either be automatic or manual assignments of particular images to locations. These locations can also be changed 
and saved while using PDH. Figures 7 - 9 show four contexts with different views of the timeline of our lab's history. When the users of PDH load one of these contexts, the documents they are viewing are displayed as thumbnails in the appropriate position on the background image, based on the time associated with the document. Users can pan and zoom on these background contexts. Contexts can also be used to define subjective views of geography, to organize documents conceptually (e.g., by research area), or to provide a backdrop for storytelling.

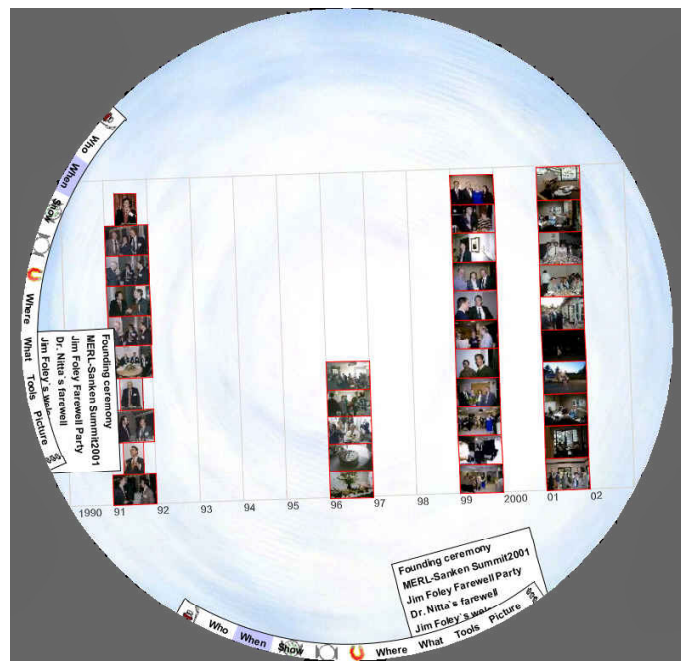

Figure 6: A linear calendar personal view of the lab history timeline.

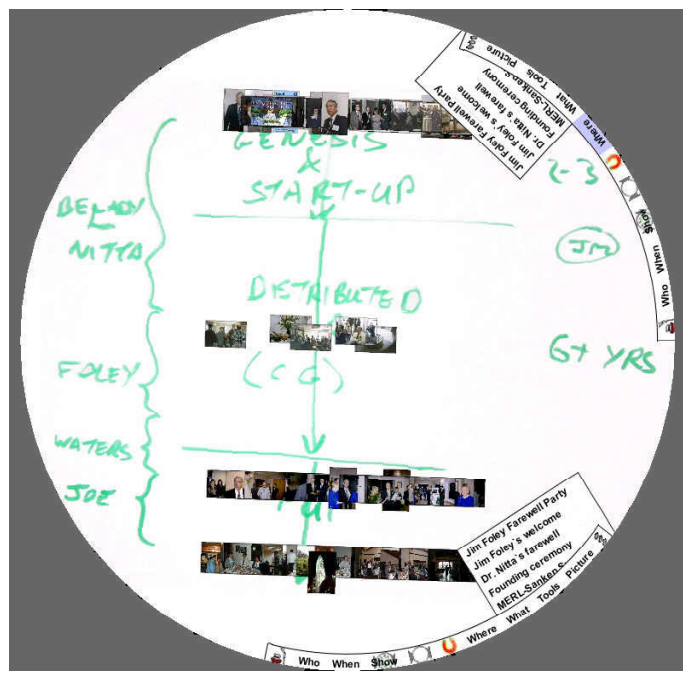

Figure 7: Four personal views of the lab history timeline.

\section{Association Generation and Presentation}

We have designed some functions of PDH to support the serendipitous discovery of information, which we call associations, related to what the users are currently viewing or have recently viewed. Associations are unobtrusive reminders of related events to the group of users. These pictures are one way in which computers can add value to experience sharing by suggesting related information, either from the users personal information or from more public sources (e.g., the web), that might be related to the context and content that are specifically requested by the user.

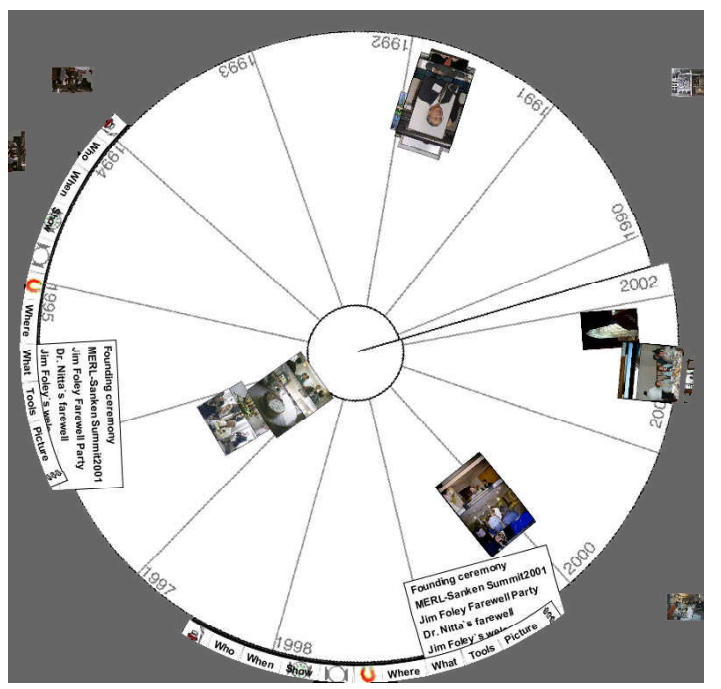

Figure 8: Four personal views of the lab history timeline.

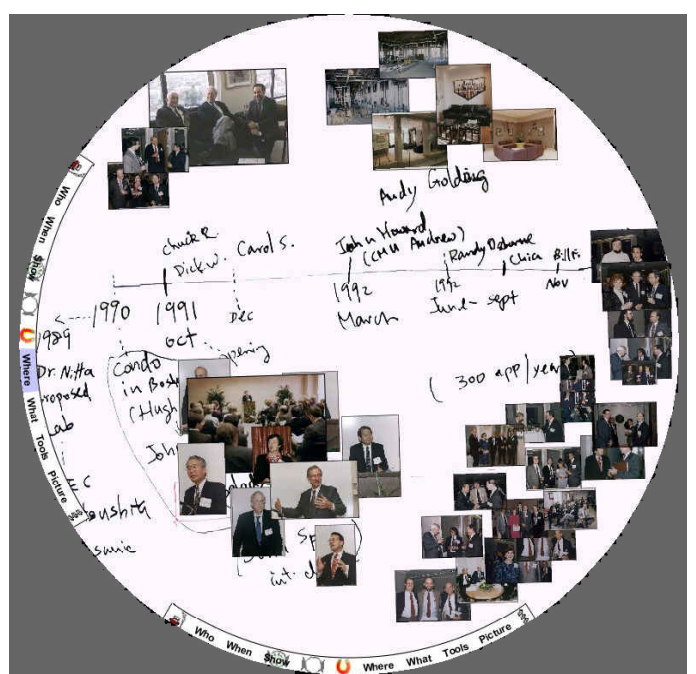

Figure 9: Four personal views of the lab history timeline.

We have both passive and active mechanisms for generating associations. Passive associations are displayed around the perimeter of the circular display (although users can turn on or off this feature). Currently the ring of pictures is static, as shown in Figure 10, though we envision a slowly moving stream of pictures moving around the perimeter. In our current system, the passive associations are generated according to the set of pictures on the table. They correspond to the result of a disjunctive variation of the query that was used to retrieve the images on the table. By replacing the AND constraint with an OR constraint, we obtain related images in the resulting relaxed query. Since the images are often grouped by events and/or time which does not provide the diversity of data we would like to see 
in the association ring, we need to post process the set of resulting images. First we remove the images already on the table from the relaxed query results. The second process randomly permutes the order of elements to present a representative set of images instead of just displaying the first $\mathrm{N}$ images returned by the database.

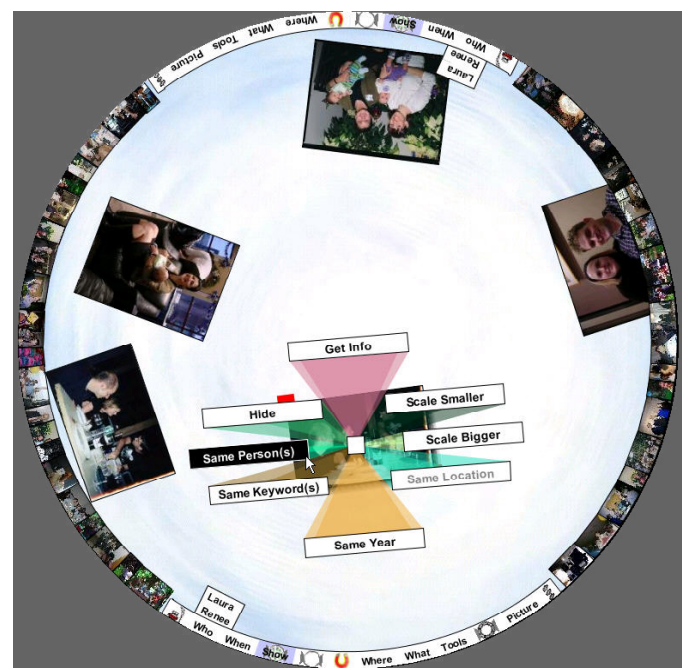

Figure 10: Free Space view with Association generation.

As shown in Figure 10, the users can also actively request documents associated with a picture in the display. When the user performs a long click on an image, then a menu pops up which allows the users to easily request more pictures that are similar along any of the who, what, when, or where dimensions. Although we currently use a simple method to retrieve the requested pictures, we plan to use the context generated by the recent activity of the users to guide the decision of which pictures to retrieve. Active associations were very popular in the user evaluation described in Section 5.

\section{Multi-level Annotation}

To facilitate the building and sharing of group histories, PDH system provides an annotation tool. It supports collaborative and continuous history building using photos and documents. We use a central database for collections of history data. The annotation tool facilitates anytime anywhere annotation. Any lab member can annotate the photos/data he/she captured/collected, and then save the data and annotation in our central repository.

Many of today's digital photo organizers and digital libraries allow the user to annotate each photo or document. Although this level of annotation is useful when viewing individual or collections of documents and photos, it is insufficient for capturing group histories. Group histories embody information about the group and about the individuals in the group, not just about the documents in the database. For example, we wanted to record information about the members of the lab, such as when he/she joined the lab, and where he/she graduated from. For a lab's history, the user may want to talk about the growth of the lab through the years; for a family to share its history, one may want to see the family tree or where have all the family members lived in the past decade. These types of information are not about individual documents.

A related problem that we experienced during the initial development of PDH was that the system often displayed an entire class of documents that the user was not interested to see. For example, if the user selected a person and pressed the Show button, the technical reports (TR) the person had written would dominate the screen display area.

To address these limitations, PDH offers a set of new annotation types and interface mechanisms. We created 3 document types for the lab history content - Photo, LabMember and TR. The type Photo associates the annotations entered by a user with the particular photo being annotated while the types LabMember and TR associate the annotation with a representative portrait of a lab member or a representative image of a technical report respectively. The user chooses a representative image from the complete collection. For example, we have annotated each researcher and administration staff in our lab as a LabMember type including his/her hiring date, and where he/she attended school. With these different types of annotation, the user of PDH can display how many technical reports the lab has produced in a particular year, or how many people joined or left the lab during a specific time period. Figure 11 shows the lab's growth in personnel over the past 10 years.

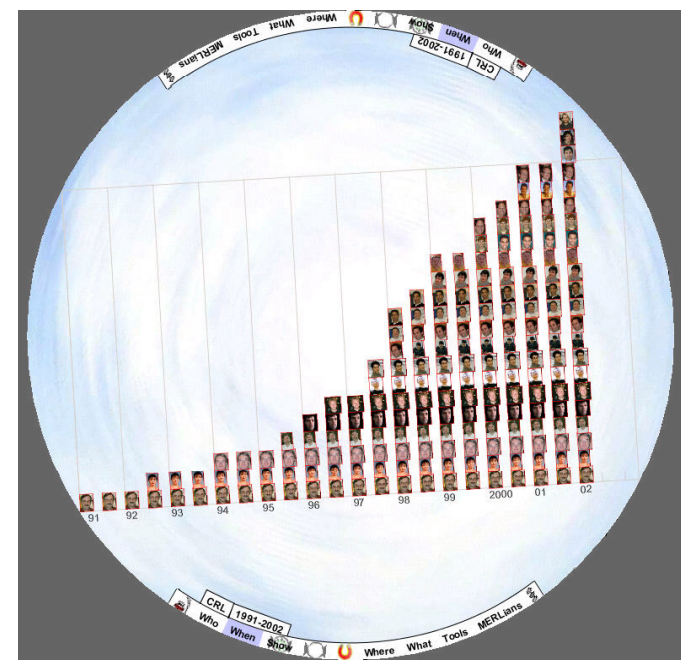

Figure 11: The lab's growth.

\section{USER STUDY}

Evaluating $\mathrm{PDH}$ is a challenge because it contains many novel features and because there are no clear performance metrics for how much a system enhances conversation and supports experience sharing. While we are interested in whether each feature is usable and easy to learn, we thought it more important to first evaluate the overall suitability of the PDH system as an interface for sharing group histories. The goal of our initial and formative user study was to see 
if people could use the system to tell stories and to discover information of interest to them by navigating through the data in the group history.

\section{Methods}

Our study's participants consisted of seven pairs of employees from our lab, including both administrative and research staff. Participants were paired with individuals whom they interacted with on a regular basis. Only one of the 14 users had prior experience with PDH. Additionally, the experimenter was a user study consultant not on the PDH project team at the time (and then subsequently joined the project team). Both users of each pair were given their own stylus and asked to operate the interface collectively.

Each session lasted about 90 minutes and was videotaped. The sessions began with a 20-minute tutorial given by the experimenter. The tutorial walked the participants through a subset of PDH's functionality using a set of pictures of famous people in famous places.

After the tutorial, the experimenter loaded in the group history we had constructed (containing 1000 images of people and events, and 250 of Technical Reports). Participants were asked to "explore the system, look at the pictures, and talk to one another." The experimenter remained near the table in order to answer questions about the interface while the pair used the system. After 30 to 50 minutes of free exploration, the experimenter asked the pair to compose a story about one of the past directors of the lab. Pairs were given ten minutes to construct a story, after which they presented their story to the experimenter.

After presenting their story to the experimenter, each user was asked to fill out their own questionnaire. (three of the subjects were unable to finish their questionnaires due to time constraints) The questionnaire started by asking the user to list the best three things about the interface as well as the worst three things. The questionnaire then listed a series of 19 statements. The user was asked to indicate how strongly they agreed with each statement by circling a number between one and seven. One indicated that they "strongly disagreed" with the statement, and seven indicated that they "strongly agreed" with the statement. The results of these questionnaires are listed in Table 1.

The questionnaire also asked the participants to estimate the percent of time they and their partner controlled the interface as well as a few other questions specific to the design of PDH that are discussed later in this section.
Table 1: This table summarizes the users' responses to the statements in our questionnaire. Low average rank indicates user's concern with the statement.

\begin{tabular}{|l|c|c|}
\hline & $\begin{array}{c}\text { Avg. } \\
\text { Rank }\end{array}$ & $\begin{array}{c}\text { Rank } \\
\text { SD }\end{array}$ \\
\hline $\begin{array}{l}\text { I was never confused about why the PDH was doing } \\
\text { certain things. }\end{array}$ & 3.73 & 2.50 \\
\hline I never got confused about the state of a search. & 4.72 & 2.71 \\
\hline I always knew exactly how to do what I wanted to do. & 6.63 & 3.49 \\
\hline $\begin{array}{l}\text { Resizing pictures to the size I wanted them was very } \\
\text { easy to do. }\end{array}$ & 7.54 & 3.64 \\
\hline I always knew exactly where I was in the interface. & 7.72 & 2.93 \\
\hline I had no problems finding the images I wanted. & 8.09 & 3.28 \\
\hline $\begin{array}{l}\text { I found opening and closing groups of people very } \\
\text { easy to do. }\end{array}$ & 8.36 & 5.47 \\
\hline $\begin{array}{l}\text { Selecting events and things in the WHAT view was } \\
\text { very easy to do. }\end{array}$ & 8.45 & 2.46 \\
\hline Rotating the table was very easy to do. & 8.63 & 5.97 \\
\hline $\begin{array}{l}\text { I was confused about why the bookmarks took me } \\
\text { back to the state they did. }\end{array}$ & 8.81 & 7.08 \\
\hline $\begin{array}{l}\text { Selecting a time period in the WHEN view was very } \\
\text { easy to do. }\end{array}$ & 8.81 & 6.11 \\
\hline The pop-up menus were very easy to use. & 9.00 & 5.51 \\
\hline $\begin{array}{l}\text { I found laying out people in the WHO view very easy } \\
\text { to do. }\end{array}$ & 9.27 & 5.19 \\
\hline I was able to tell the story I wanted to. & 9.36 & 6.23 \\
\hline $\begin{array}{l}\text { Selecting people and groups of people in the WHO } \\
\text { view was very easy to do. }\end{array}$ & 9.45 & 5.75 \\
\hline $\begin{array}{l}\text { I was always certain why specific images were visible } \\
\text { in the SHOW view. }\end{array}$ & 9.81 & 5.53 \\
\hline $\begin{array}{l}\text { Removing some constraints from a search was easy to } \\
\text { do. }\end{array}$ & 10.54 & 6.09 \\
\hline Switching between views was very easy to do. & 11.72 & 3.74 \\
\hline Moving pictures around the table is very easy to do. & 11.81 & 4.94 \\
\hline
\end{tabular}

\section{Results}

\section{General Observations}

Our preliminary findings were encouraging. We were gratified that the users seemed to have fun, laugh a lot, and actively interact with each other as well as the data. In each session, at least one user told the other something new about a person or event in the photographs. In all sessions, the users taught the experimenter (who was new to the lab) something about the lab based on the digital content of the group history. Although the associations were not in the tutorial, most pairs found the active associations and used them extensively. All the users were able to quickly perform searches after the initial tutorial. Everyone looked for pictures of himself or herself. Indeed, about half of the users were disappointed at how few pictures there were of them. While the questionnaires were generally positive, the 
users pointed out many limitations of the current system and made suggestions for improvements and additional features. For all seven groups, conversation focused around the content of the images and not on the interface. When one user ran into trouble with the interface, it was not uncommon to see the other user step in and help him/her out.

\section{Task Completion}

Five of the seven pairs were able to form and present their 'assigned' story. One pair thought they could with more time. One pair felt they could not form a good story with the current interface and data, they wanted a sequential set of bookmarks. All stories were told using multiple views. Users navigated among these views using both bookmarks and the $4 \mathrm{Ws}$. Some pairs structured their stories by time, others by events, and still others by people.

\section{Bookmarking}

All the users were able to use bookmarks to return to a previous state of the system. About half the people mentioned they would like to be able to delete a bookmark. (About half of the groups formed a bookmark accidentally.) Many users wanted the ability to merge new images into the contents of an existing bookmark. And one pair suggested that the bookmarks be a snapshot of the entire tabletop rather than a single picture within it.

\section{Discovering Information Using the 4 Ws}

The 4 Ws helped users discover surprising information that would be difficult to detect with more traditional systems. For example, two pairs selected the past director, switched to the When view, and noticed that one picture was positioned four years before the rest on the time line. They learned that he was a guest speaker at the founding ceremony of the lab, years before his employment. Similarly, another pair noticed that one person's pictures preceded her employment - her spouse was already working at the lab.

\section{Visible State}

After the tutorial, all seven groups were able to use the system to perform simple searches. Few users had trouble recognizing the state of a search within a W. For example, if users selected two people in the Who view, they expected to see pictures including either person. The relationship among views was more confusing. If they selected a person and a time range in the When view, they did not expect to see pictures that both had that person and were taken during that time range. One possible explanation for this discrepancy is that the system provides a visible cue (the list of selections) for the state of the search within a view but provides no cue for the Boolean relationship among groups. Despite the confusion, the system's AND/OR semantics seem to provide the right search functionality. For example, it is more interesting to see pictures of a person taken during a specific time than to see pictures that either contain a person or were taken during a specific time.

\section{Controlling the Application}

Virtually every subject ran into trouble at one point or another with our pen based input device. Mis-registration and occlusion of the detectors lead to missed steps and negative training. Several of the GUI control targets (most noticeably, the controls for resizing an image) were too small for the accuracy these pens allowed. As a result of this study, we are changing our input device to a touch based input device [4].

\section{Overloading Input}

About half of the users had trouble at least one time distinguishing dragging an image from opening its pop-up menu. Because a click-and-drag action moves an image and a click-and-dwell action opens the pop-up menu on an image, this confusion seems understandable. Lag coming from the ultrasound pen based input device may have compounded this problem.

\section{Visible Commands}

By far the most common feature used was the resizing of images. Users commonly selected an image to view and immediately resized the image as large as the table would allow. Oftentimes, they would hide the image rather than scale it back to its original size. This feature was so popular, that PDH now includes a "resize and get information on" button at the corner of the selected image. In the same way the resize handles provide the user with a visible interaction location, the information button now makes the command "get info" visible and easily accessible.

\section{Best and Worst Things}

Five of the eleven users who filled out a questionnaire indicated that the ability to resize and orient images was one of the best three things in the system. Two users (from different groups) listed the interaction between two people as one of the best three things. Two users said that they had fun because of the comfortable setting of the interaction. Another two users said that the large display that allowed them to collaborate well with others was one of the best parts of the system.

Users had little trouble moving pictures around the tabletop and had little trouble rotating the tabletop as a whole. However, users pointed toward difficulties resizing images as one of the hardest parts of using the system. As we observed that this was one of the most popular features in the system, this frustration seems understandable. Opening and closing large groups of people was also troublesome for our users. Several users suggested methods for helping with the layout of the many photographs contained within a group and pointed to "clutter" and "over crowding" as major problems with the system.

\section{Simultaneous Input}

We were concerned that our input mechanism, which only accepted one stylus input at a time, would create conflicts in operating the tabletop by multiple users. However, we were 
surprised that only one pair mentioned "not being able to use both stylus at once" as a problem. At the beginning of the tests, all of the pairs initially expected to each be able to manipulate digital documents simultaneously, just like real documents on a tabletop; however, the pairs quickly learned to work around this limitation and seem to have accepted it by the end of the session.

\section{Sharing Control}

In all sessions, both users participated significantly in the control of the interface. In the questionnaire, users estimated that they controlled the interface an average of $53 \%$ of the time, and that their partner controlled the interface an average of $47 \%$ of the time. The perceived difference between the percent of time a user controlled the interface and the percent their partner controlled the interface averaged $20 \%$. It was not uncommon to see the users jointly operate an interface component. For example, one user might open a menu and the other selects an item from it. The majority of subjects strongly disagreed with the statement "I had trouble distinguishing between what I was controlling and what my partner was controlling."

\section{Related Work}

There has been a lot of literature on storytelling with digital photos $[1,3]$. This literature has served as the basis for some of our conceptual design of the PDH interface. Research into digital user interface tools to support the use of digital photos for story sharing has just started to emerge [2, 9]. In PhotoFinder [9], a new utility called StoryStarter allows users to take a set of photos that have already been annotated and publish them on web pages. StoryTrack [2] is a prototype handheld device that can support in-person copresent story sharing with digital photographs. Unlike PDH, neither PhotoFinder nor StoryTrack are designed to support small groups of people to browse, collaboratively, through large collections of recordings of their lives.

Another area of related research is the large body of work on various forms of graphical and direct manipulation query interfaces, as well as attribute-based and Content-Based Information Retrieval (CBIR) for document and multimedia databases $[5,6,7,0,14,17,18]$. Although many of the techniques in these previous works are similar in spirit with our research, unlike PDH, most of these systems (except [7]) are designed to support interaction with large databases of data with which the users are unfamiliar. Moreover, they are designed for targeted information seeking, rather than support conversational exploration and browsing.

In general, almost all research in how to support experience sharing with digital data suffers from the limitations of current display technology. Indeed, while digital photographs are easier to share remotely than physical photographs, they are much more difficult to use in face-toface conversational settings. Desktop computers force people to face the direction of the screen, which often is not natural in a conversational setting. Handheld devices can only support the simultaneous viewing of the same photo by at most two people comfortably side-by-side, and the size of the screen only allows a few images to be clearly viewable at once. Motivated by these considerations, our decision is to use a tabletop display. The document orientation, visualization and layout issues brought about by such a circular display surface have been discussed in [22].

In the past few years, there has been a proliferation of beyond-the-desktop research projects, looking at how to integrate the design of computation into architectural spaces and furniture, including tabletops. The Living Memory [10] and the i-Land project [21] are two such examples. The coffee table design in the Living Memory (LiMe) project [10] employs two semi-circle tabletop displays on each coffee table. The intended application of the LiMe table is to promote the sharing of knowledge and experience among people in local communities or neighborhoods. They study how to build a collective digital living memory, while PDH focuses on the co-present sharing and exploration of the group history. The InteracTable in the $\mathrm{i}$-Land project supplies a rectangular surface to be shared among multiple users for office work environment.

$\mathrm{PDH}$ is also part of the recent research effort in the area of SDG (Single Display Groupware) [8,19,20]. Research on SDG investigates both the technology of how to support multiple-user co-present use of a shared computer display and how this technology affects groups to collaborate and interact in work, learning and play environments. In $\mathrm{PDH}$, the user interface technology support for SDG is reported in [22], including multiple control panels for multiple users, easy re-orientation of documents on the tabletop and visualization techniques for shared and private viewing of documents. The current paper contributes to the exploration of new user interface features for content browsing, story sharing and user behavior study. The three outcome variables - task performance, user reactions and group member relations - that were described in [11] provided guidelines for the user study we carried out in this paper.

\section{Conclusion and Future Directions}

This paper reports on the first application of the Personal Digital Historian project. We developed, used, and evaluated systems for building and sharing a group history of our research lab. Our main finding was the (preliminary but encouraging) validation of the suitability of the $\mathrm{PDH}$ interface for sharing group histories. We also presented several novel extensions to $\mathrm{PDH}$, including visual bookmarking, passive and active associations, and the integration of background contexts.

Our future work includes addressing many of the limitations pointed out by our users. We plan to consider how to support multiple users' turn taking and simultaneous interaction at both the device and software level. Finally, we will perform larger and longitudinal studies to more fully evaluate the strengths and weaknesses of PDH for supporting experience sharing in general and group histories in particular. 


\section{REFERENCES}

1. Akeret, R.U., Photolanguage,: How Photos Reveal the Fascinating Stories of Our Lives and Relationships, W. W. Norton \& Company, Inc., 500 Fifth Avenue, New York, N.Y. 10110. (2000).

2. Balabanovic, M., Chu, L., Wolff, G., Storytelling with Digital Photographs, in Proceedings of CHI 2000, (The Hague The Netherlands, April, 2000), 564-571.

3. Chalfen, R., Snapshot: Versions of Life, Bowling Green State University Popular Press, Bowling Green, $\mathrm{OH}$ 43403, 1987.

4. Dietz, P., Leigh, D., DiamondTouch: A Multi-User Touch Technology, in Proceedings of ACM UIST'01, (Orlando FL, November 2001), 219-226.

5. Digital Library Project, University of California, Berkeley, http://elib.cs.berkley.edu.

6. Hibino, S., Rundensteiner, E.A., Processing Incremental Multidimensional Range Queries in a Direct Manipulation Visual Query Environment, in Proceedings of ICDE'98. (Orlando FL, February 1998), 458-465.

7. Dourish, P., Edwards, W.K., LaMarca, A., Salisbury, M., Presto: An Experimental Architecture for Fluid Interactive Document Spaces, ACM Transactions on Computer-Human Interaction, Vol. 6, No 2, 133-161, 1999.

8. Inkpen, K., Booth, K.S., Klawe,M.,, McGrenere,J., The Effect of Turn-Taking Protocols on Children's Learning in Mouse-Driven Collaborative Environments, in Proceedings of GI'97 (Kelowna BC, May 1997), 138145.

9. Kang, H., Shneiderman, B., Visualization Methods for Personal Photo Collections: Browsing and Searching in the PhotoFinder, in Proceedings of ICME'2000, (New York NY), 1539-1542.

10.Kyffin S., The LiME Project, Philips brochure published at: http://www.design.philips.com/lime/ download/brochure.pdf

11.McGrath, J.E., Hollingshead, A.B., Groups Interacting With Technology, Sage. 1994.

12. Mimio, ultrasonic position capture system http://www.mimio.com/meet/mimiomouse.
13.Platt, J., AutoAlbum: Clustering Digital Photographs Using Probabilistic Model Merging, In Proceedings of IEEE Workshop on Content-based Access of Image and Video Libraries (CBAIVL'2000, Hilton Head SC, June 2000), 96-100.

14. Santini, S., Jain, R., Integrated Browsing and Querying for Image Databases, IEEE Multimedia, Vol. 7, No. 3, July-September 2000, 26-39.

15. Schilit, B.N., Golovchinsky, G., Price,M.N., Beyond Paper: Supporting Active Reading with Free Form Digital Ink Annotations, in Proceeding of ACM CHI'98, (Los Angeles CA, April 1998), 18-23.

16. Shen, C.; Lesh, N.B.; Moghaddam, B.; Beardsley, P.A.; Bardsley, R.S., Personal Digital Historian: User Interface Design, in Proceedings of Extended Abstract of CHI'2001 (Seattle WA, April 2001), 29-30.

17. Shin, D-G., Nori, R. S., CBM: A Visual Query Interface Model based on Annotated Cartoon Diagrams, in Proceedings of the Working Conference on Advanced Visual Interfaces (AVI'2000, Palermo Italy, May 2000), 242-245.

18. Shneiderman,B., Visual User interfaces for Information Exploration, in Proceedings of the $54^{\text {th }}$ Annual Meeting of The American Society for Information Sciences, Vol. 28 (Washington DC, October 1991), 379-384.

19. Stewart, J., Bederson, B., Druin, A., Single Display Groupware: A Model for Co-present Collaboration, in Proceedings of CHI'99 (Pittsburgh PA, May 1999), 286-293.

20. Shoemaker, G.B.D., Inkpen, K.M., Single Display Privacyware: Augmenting Public Displays with Private Information, in Proceedings of CHI'2001, (Seattle WA, March-April 2001), 522-529.

21. Streitz, N. et al., i-LAND: An interactive Landscape for Creativitiy and Innovation, in Proceedings of CHI '99 (Pittsburgh PA, May 1999), 120-127.

22.Vernier, F., Lesh, N.B. and Shen, C., Visualization Techniques for Circular Tabletop Interfaces, in Proceedings of AVI'2002 (Trento Italy, May 2002), 257-266.

23.U.S. Department of Education, Office of Educational Research and Improvement, Helping Your Child Learn History, http://www.ed.gov/pubs/parents/History/. 